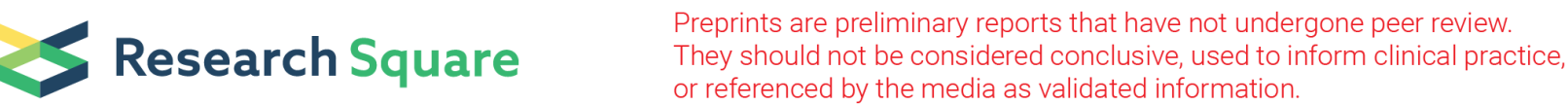

\section{Absorbable self-retaining barbed double-needle suture(SRBDS) in transperitoneal laparoscopic partial nephrectomy for T1 renal neoplasms}

Hai-bin Wei

Zhejiang Provincial People's Hospital

Yu-chen Bai

Zhejiang Provincial People's Hospital

Qi Zhang

Zhejiang Provincial People's Hospital

Zu-jie Mao

Zhejiang Provincial People's Hospital

En-hui Li

Zhejiang Provincial People's Hospital

\section{Shuai Wang}

Zhejiang Provincial People's Hospital

Xiao-long Qi

Zhejiang Provincial People's Hospital

Feng Liu

Zhejiang Provincial People's Hospital

DaHong Zhang ( $\nabla$ zhangdahong168@163.com )

Zhejiang Provincial People's Hospital

\section{Technical advance}

Keywords: Laparoscopic partial nephrectomy, nephron-sparing surgery, kidney neoplasm, self-retaining barbed suture

Posted Date: October 29th, 2020

DOI: https://doi.org/10.21203/rs.3.rs-97671/v1

License: (c) (1) This work is licensed under a Creative Commons Attribution 4.0 International License.

Read Full License 


\section{Abstract}

\section{Purpose}

The purpose of study was to evaluate the safety and hemostatic efficacy of absorbable self-retaining barbed double-needle suture (SRBDS) in transperitoneal LPN for T1 renal neoplasms.

\section{Methods}

In total, 75 patients with renal neoplasm masses $(<7 \mathrm{~cm})$ undergoing transperitoneal LPN were collected since January 2018 to October 2019. After tumor resection with a cold Endoscissor, 3-0 and 0 SRBDS was performed for inner and outer running stitches without either hem-o-lok or LapraTy clip, respectively. Patient demographic information, neoplasm characteristics, perioperative factors, and oncologic outcomes were collected and analyzed.

Results

According to our result, Median tumor size and R.E.N.A.L score was $4.69 \mathrm{~cm}$ and 7. Median operative time and overall warm ischemia time was $73 \mathrm{~min}$ and $13.14 \mathrm{~min}$. Mean estimated blood loss was $67 \mathrm{ml}$. intraoperative complications was not showing up and no case was switched to open radical nephrectomy. Postoperative pathological assessment confirmed that negative surgical margins. only single patient experienced secondary surgery-related hemorrhage on the fifth day post-operation, and was treated by transfusion and digital subtraction angiography (DSA) highly selective embolization of the bleeding vessel. No patients developed urinoma or urinary fistula. the overall complications were manageable during three-year follow-up period.

Conclusion

SRBDS was a safe and efficient surgical endoscopic suturing technique with low complication rate in T1 renal neoplasms, which might be considered as an alternative to other suturing techniques, tissue sealants and glues for partial nephrectomy in the future.

\section{Background}

Small asymptomatic renal masses are increasingly being detected due to the ultrasonography and CT scans as common medical examination or diagnostic procedures. Partial nephrectomy has been generally treated as the first-line for such conditions [1, 2]. Partial nephrectomy could provide oncologic outcomes compared to radical nephrectomy with maximal preservation of unaffected nephrons [3]. Since the introduction of partial nephrectomy, minimally invasive partial nephrectomy such as laparoscopic partial nephrectomy (LPN) and Da Vinci-Assisted robotic partial nephrectomy has became increasingly important and more common in the field of renal neoplasms. both of them became more acceptance. 
Both LPN and Da Vinci-Assisted robotic partial nephrectomy are reserved exclusively for skilled laparoscopists due to complicated technical. Intracorporeal suturing for hemostasis and nephrorrhaphy, which including renal parenchymal and pelvicalyceal repairing or closure. This procedure was still an challenge and time-consuming procedure due to limited warm ischemia time (WIT) [4, 5]. Therefore, there is a surge in suturing alternatives for hemostasis and nephrorrhaphy in LPN surgery, seen in clinical practice $[2,6-20]$.

SRBDS was an advanced surgical suture which consists of delayed-absorbable suture material and two needles for suturing in different directions. It could decreases the possibility of slippage through renal tissue and avoids the use of clips and knots. However, this procedure could relatively increases efficiency and make WIT been shorter than other procedure [6]. There are few studies was reported which concerning the potential of SRBDS in LPN. Hence, we performed SRBDS in LPN, and investigated the safety and hemostatic efficacy of SRBDS in transperitoneal LPN for T1 renal neoplasms by assessing the perioperative conditions and analyzed three-year follow-up outcomes of 75 patients.

\section{Materials And Methods}

\section{Patients}

This study was collected at the Department of Urology, Zhejiang Provincial People's Hospital, People's Hospital of Hangzhou Medical College, Hangzhou, Zhejiang, China. A total of 75 patients with unilateral small renal masses $(<7 \mathrm{~cm})$ were included in our clinical trial since January 2018 to October 2019.All surgical procedures were performed by 3 experienced surgeons, who had successfully treated more than 100 prior cases with those procedures.

Inclusion criteria for patients were T1 renal neoplasms suitable for LPN, i.e. unilateral masses smaller than $7 \mathrm{~cm}$ in diameter indicated by imaging examinations such as ultrasonography, CT scan or MRl; Normal contralateral kidney and without metastatic disease.

Exclusion criteria including patients who older than 75 years with only one functional kidney, and requiring additional surgical interventions on the kidney. And more, Patients who was intaking anticoagulant or anti-aggregate should be exclude as well.

\section{Surgical procedure}

First at all, the patients were secured in flank position with lateral tilt. The Veress needle technique was performed in order to establish $\mathrm{CO}_{2}$ pneumoperitoneum with $12-15 \mathrm{mmHg}$, which should be performed at lateral of rectus muscle with level of the umbilicus. and then a 10-mm laparoscopic camera port was set at the same position; other port was set with $30^{\circ}$ lens and looking at upward.A $12-\mathrm{mm}$ port was set with $3 \mathrm{~cm}$ above the umbilicus at the anterior axillary line. Finallly, two 5-mm ports were inserted at the mid-clavicle and at the posterior axillary line below the 12th rib costochondral margin, respectively. 
The camera port was set by excellent vision at the renal hilar anatomy, and the standard technique was performed to expose the whole kidney. For right-sided neoplasms, the liver was retracted anteriorly and cephalad; For left-sided neoplasms, the spleen and pancreas were reflected medially. For endophytic neoplasms, intraoperative ultrasonography was generally used to confirm the resection margin. The Gerota fascia was dissected off from the surface of the kidney, but the perirenal fat intact over the neoplasms was reserved for subsequent grasping and resecting.

The renal artery was entirely separated and clamped individually by the laparoscopic bulldog clamp, and the warm ischemia time was noted. The preserved perirenal fat close to the tumor was grasped and the tumor was elevated away from the tumor bed on the counter-traction. The renal parenchyma was resected with cold Endoscissor, and intraoperative frozen pathology was required to confirm negative margins.

The suturing process was divided into two layers, i.e., the inner and the outer layers. At first, the inner layer was divided into two parts from the middle, where the SRBDS (Stratafix spiral, Johnson \& Johnson medical companies, China) was locked and changed direction. A 3 - 0 SRBS was equipped with bidirectional barbs of $14 \times 14 \mathrm{~cm}$ and two needles, one on each end. We generally used $3-0$ SRBS for the inner layer and 0-0 SRBS for the outer layer. One needle was maneuvered from the outside of the deep tumor bed through the tumor bed, and brought out from the other side of the tumor bed. This needle ran from the middle throughout the tumor bed to the end. The other needle sutured from the middle to the end of other tumor bed.and the last suture was tightened. In addition, the renal pelvis or calices were also closed by this running suture. Subsequently, suture of the outer layer should be performed after removing the bulldog.which could relative reduced the WIT. The suture procedure was repeated for the outer layer, as shown in Fig. 1. if there is bleeding which could be identified after suturing of the outer layers. An extra suture by figure-of-eight would be added in cases where bleeding was observed. Finally, all suture lines were cut off and the needles were taken out. Chenk the process of nephrorrhaphy, no hemostatic agents, glues, hem-o-lock clips, LARPA-TY clips, or knots were used.

\section{Measurements and data analysis}

All patient-related data were collected and analyzed perioperatively, including age, sex, tumor characteristics (size, left/right, location and pathology), and surgical information such as operative time, WIT, suture time, estimated blood loss and hospitalization, perioperative complications and prognosis of renal function. Continuous variables were generally expressed as mean \pm standard deviation (SD), whereas the continuous variables not fitting a normal distribution were reported as median and range.

\section{Results}

Patients data and perioperative characteristics are listed in Table 1. All 75 transperitoneal LPN procedures were successfully performed. Mean suture time and WIT were $8.10 \mathrm{~min}$ and $13.14 \mathrm{~min}$, respectively. Estimated blood loss per person was $67 \mathrm{ml}$, which was in the controllable range. 
Table 1

Perioperative patients' characteristics

\begin{tabular}{ll} 
Perioperative & $\begin{array}{l}\text { SRBS }(\mathbf{n}=75) \\
\text { Mean } \pm \text { SD or }\end{array}$ \\
\hline characteristic & $51(33-75)$ \\
\hline Sex (m/f) & $42 / 33(-)$ \\
\hline R.E.N.A.L score & $7(5-9)$ \\
\hline Suture time (min) & $8.10 \pm 2.16$ \\
\hline WIT (min ) & $13.14 \pm 5.81$ \\
\hline Operative time ( min ) & $73(61-94)$ \\
\hline Blood loss ( $\mathrm{ml})$ & $67 \pm 15.81$ \\
\hline Hospital stay (d) & $7(5-10)$
\end{tabular}

SRBS, self-retaining barbed double-needle suture; SD, standard deviation; $\mathrm{m} / \mathrm{f}$, male/female; WIT, warm ischemia time.

Table 2 summarizes the tumor characteristics. The resected masses ranged from $3.3 \mathrm{~cm}$ to $6.8 \mathrm{~cm}$, and $85.3 \%$ were confirmed malignant by pathological assessment. In addition, final pathologic characteristics was showing up the all surgical margins were negative, which confirmed the success of the resection.

Table 2

Tumour characteristics of 75 cases

\begin{tabular}{|ll|}
\hline Tumour characteristics & SRBS $(\mathbf{n}=\mathbf{7 5})$ \\
\hline Size (range) & $4.69(3.3-6.8)$ \\
\hline Right/left & $35 / 40(-)$ \\
\hline Location (upper/middle/lower) & $35 / 10 / 30$ \\
\hline Tumour histology & $61(81.3 \%)$ \\
\hline Renal clear cell carcinoma & $11(14.7 \%)$ \\
\hline Angiomyolipoma & $3(4.0 \%)$ \\
\hline Renal chromophobe cell carcinoma & \\
\hline SRBS, self-retaining barbed double-needle suture; SD, standard deviation. \\
\hline
\end{tabular}

The incidence of perioperative complications was very low. No case was switched to open radical nephrectomy.There was one patient suffering from secondary surgery-related hemorrhage, who 
underwent transfusion and DSA highly selective angioembolization of the bleeding arterial branch. One patient presented with postoperative fever $\left(>38.5^{\circ} \mathrm{C}\right)$, but responded well to intravenous antibiotic treatment. No other perioperative complications, such as atelectasis, fistula, delayed hemorrhage, extraction-site hernias and urinary fistula were observed. During the one-year follow-up, no patient suffered from renal insufficiency, tumor recurrence or distant metastasis.

We have reviewed trials on SRBDS including LPN and open partial nephrectomy, published over the past 3 years, and listed the outcome data in Table $3[4,6,8,21]$. Obviously, SRBDS was as safe and hemostatically efficient as reported in the previous studies. As evidenced by the data, the procedure was associated with only few manageable complications. 
Table 3

Summary of outcome data about the other studies of the unidirectional barbed suture

\begin{tabular}{|c|c|c|c|c|c|}
\hline & $\begin{array}{l}\text { Our study- } \\
2019\end{array}$ & $\begin{array}{l}\text { Wahafu- } \\
2018\end{array}$ & $\begin{array}{l}\text { Wang- } \\
2018\end{array}$ & $\begin{array}{l}\text { Schauer- } \\
2018\end{array}$ & Jeon-201c \\
\hline Patient number & 75 & 298 & 36 & 15 & 13 \\
\hline Follow-up terms & 12 months & 28 months & 11 months & - & - \\
\hline Suture type & SRBDS & Monocryl & Quill SRS & V-Loc SRBS & V-Loc \\
\hline \multicolumn{6}{|c|}{ Perioperative characteristic } \\
\hline Tumor Size $(\mathrm{cm})$ & 4.69 & 3.41 & 3.1 & 3.5 & 2.5 \\
\hline Suture time (min) & 8.10 & - & 10.4 & - & - \\
\hline WIT (min) & 13.14 & 16.55 & 15.2 & 15.2 & 24.5 \\
\hline $\begin{array}{l}\text { Operative time } \\
\text { (min) }\end{array}$ & 73 & 74.34 & 78.5 & - & 248.5 \\
\hline Blood loss (ml) & 67 & 43.44 & 60.5 & - & 319.2 \\
\hline Hospital stay (d) & 7 & 6.19 & 5.9 & - & 3.8 \\
\hline \multicolumn{6}{|l|}{ Complications } \\
\hline $\begin{array}{l}\text { Perirenal } \\
\text { hematoma }\end{array}$ & $0 \%$ & $0 \%$ & - & $0 \%$ & - \\
\hline Haemorrhage & $1.3 \%$ & $0.7 \%$ & $2.8 \%$ & - & - \\
\hline Transfusion & $1.3 \%$ & $0.7 \%$ & $0 \%$ & $0 \%$ & $15.4 \%$ \\
\hline Urine Leak & $0 \%$ & $0.3 \%$ & $0 \%$ & - & - \\
\hline Hypercarbia & $0 \%$ & $0 \%$ & - & - & - \\
\hline Embolism & $0 \%$ & $0 \%$ & - & $0 \%$ & - \\
\hline Urinoma & $0 \%$ & - & - & $6.7 \%$ & $0 \%$ \\
\hline Pyrexia & $0 \%$ & - & - & $6.7 \%$ & - \\
\hline Postoperative fever & $1.3 \%$ & - & - & $0 \%$ & - \\
\hline
\end{tabular}

\section{Discussion}

Renal cell carcinoma(RCC) is one of the most common urologic neoplasms, The incidence of male was up to $>15 / 100000$ in several Northern and Eastern European countries and among US blacks [22]. Although the stabilization of mortality trends has been achieved in many highly developed countries, RCC 
identification continues to increase worldwide, along with the popularization of imagining techniques such as ultrasound and CT scans [22, 23]. A considerable part of these tumors are primary, small and discovered incidentally, which require surgical intervention when conservative treatment fails.

The 2009 version of the American Urological Association guidelines recommends that nephron-sparing approaches, such as partial nephrectomy are appropriate for clinical stage one $(<7 \mathrm{~cm})$ renal masses [1]. LPN is becoming increasingly standardized due to the feasibility and efficacy of laparoscopic techniques. Moreover, LPN has numerous advantages, which is better than open partial nephrectomy ,terms of blood loss, WIT, operative duration, perioperative morbidity and hospitalization. However, three shortcomings and technical difficulties of LPN have prevented it from widespread use and application. Firstly, intracorporeal suturing for hemostasis, renal parenchymal repair and closure of the pelvicalyceal, especially in the retroperitoneal route due to the relatively small space available for operation,all those performed were complicated. Therefore, technological improvement is needed to flatten the steep learning curve specific to the laparoscopic approach. Secondly, intracorporeal suturing cause WIT been longer than before. Every minute of WIT counts when the renal hilum is clamped, Hence, every additional minute is associated with a $5 \%$ increase in the risk of developing acute renal failure, and $6 \%$ increase in the risk of new-onset stage IV chronic kidney disease during the follow-up [24]. Hence, it is important to improve the suturing process and reduce the WIT. Thirdly, LPN has been associated with an increased rate of bleeding and urinary leak [25]. Suturing is the most effective method of hemostasis and preventing urinary leaks [4]. SRBDS is an advanced surgical suturing to reduce those complications.

SRBDS consists of delayed-absorbable suture material and 2 needles for different direction suture, it is a relative new method in the field of surgical suturing. There are several advantages for SRBDS. Firstly, SRBDS has barbs lining at intervals of $1.25 \mathrm{~mm}$, which could prevented slippage through "self-cinching mechanism" [4] and could free one hand from maintaining the wire tension. Therefore, SRBDS provides a feasible surgical method to decrease the learning curve of complex laparoscopic tasks, especially intracorporeal suturing. Secondly, SRBDS has two needles and can be used for suture in different directions, from the suture midpoint. In addition, SRBDS does not require the use of knotting or clips, such as Hem-o-lok and Lapra-Ty clips. At the end of the suturing process, the suture could be cut off directly. SRBDS is a method which carried out separately in the inner and outer layer by running suture, which make the suture easier and more efficient. Finally, SRBDS results in less parenchymal tearing and avoids the "cheese-slicing" because of this suture process with balanced force. which could reduce complications such as bleeding and urinary leakage [4].

In China, Wang and Wahafu have confirmed the effectiveness and safety of SRBDS in LPN $[4,6]$. In their studies, bi-directional barbed suture was used in the retroperitoneal route of LPN, and the left quill line was used to tie a knot, or a Hem-o-lock was used to close the end of the suture. In addition, Xu Zhang performed the running suture after anchoring with one Hem-o-lok clip (Weck surgical instruments) at one end, and set another anchor at the other end after suturing [4]. These approaches are quite different from our SRBDS method. For us, the transperitoneal approach is more favorable because of its larger operative space, wider separation of ports, and more familiar landmarks. Therefore, all surgical interventions were 
finished in the transperitoneal route of LPN, regardless of tumor location. Consequently, the purpose of this article was to prove that the SRBDS is an alternative method for suturing without using any knot or clip in the transperitoneal LPN.

Time and WIT was reduced if you comparing to the previous studies $[4,6]$. We removed the bulldog clamp before suturing the outer layer. because early withdrawal of the bulldog clap might reduced the WIT in the future. If we compare to traditional methods, We did not use any hemostatic agents or glues, hem-o-lock clips, LARPA-TY clips or knots through the process, and directly cut off the quill line at the end of the suture. Hence, according to our summaries, SRBDS provides an efficient and feasible choice for T1 renal neoplasms.

In our opinion, safety always are important. SRBDS slightly decreased the mean hemoglobin (estimated blood loss $67 \mathrm{ml})$, which was similar to the results obtained by Wang $(60.5 \mathrm{ml})$, but higher than the volume reported by Wahafu $(43.44 \mathrm{ml})$ (Table 3$)$. We deduced that tumor size may be responsible for these differences. The increase in tumor size is associated with longer operative time and blood lost. Meanwhile, the blood loss is estimated during surgery, and is associated to the surgeon's experience and intraoperative irrigation. Consider of the lack of clips and lower suture cost into account, SRBDS is a feasible nephrorrhaphy choice in LPN.

According to our outcome, that reveals the complications were rare and usually could be control. Actually,there were few disadvantages in our study. Follow-up was short (12 months) and the number of participants was small. The trial also lacked randomized control. In addition, glomerular filtration rate evaluated by nuclear medicine was not carried out, due to the restrictions of health care cost during the follow-up, so we failed to assess the impact of surgery on the operated kidney. These shortcomings will be addressed in a subsequent study.

Anyway, SRBDS is a method which could provide favorable outcomes with lower morbidity and complications over a one-year follow-up period.

\section{Conclusion}

SRBDS is a safe and stable endoscopic suturing technique, associated with a low complication rate in T1 renal neoplasms over a follow-up period of 12 months. It is a promising procedure that should be considered as an alternative technique for hemostasis and renography in the future. However, multicenter, larger-scale, double-blinded, and randomized controlled trials with longer regular follow-up periods are needed to confirm the durability of the surgical outcome.

\section{Abbreviations}

LPN: Laparoscopic partial nephrectomy

OPN: Open partial nephrectomy 
SRBDS: Self-retaining barbed double-needle suture

WIT: Warm ischemia time

SD: Standard deviation

RCC: Renal cell carcinoma

\section{Declarations}

\section{- Ethics approval and consent to participate}

All study participants were informed about the planned procedure and signed informed consent. The study was approved by the ethics committee of Zhejiang Provincial People's Hospital .

\section{- Consent for publication}

We have obtained written informed consent from the patients for publication of this article and all the accompanying images. A copy of the written consent is available for review by the Editor of the journal.

\section{- Availability of data and material}

The datasets used and/or analysed during the current study are available from the corresponding author on reasonable request. The authors declare that they have no restrictions of data and materials.

\section{- Competing interests}

The authors declare that they have no competing interests.

\section{- Funding}

No funding from any source for this study.

\section{- Authors' contributions}

DHZ and FL made substantial contributions to conception and design and acquisition of data. HBW and YCB has been involved in drafting the manuscript. HBW, XLQ and FL made substantial contributions to conception and design. QZ, ZJM and EHL made substantial contributions to analysis and interpretation of data. SW revised it critically for important intellectual content. All authors have read and given final approval of the version to be published.

\section{- Acknowledgements}

We would like to thank the Department of Urology, Zhejiang Provincial People's Hospital for the population and optimization of laparoscopic techniques in order to benefit patients. In addition, we would 
like to thank all the patients for giving us the permission to use the medical information for this publication.

\section{- Authors' Information.}

Department of Urology, Zhejiang Provincial People's Hospital, People's Hospital of Hangzhou Medical College, No. 158, Shangtang Road, Xiacheng District, Hangzhou 310014, Zhejiang, China.

\section{References}

1. Campbell SC, Novick AC, Arie B, et al. Guideline for management of the clinical T1 renal mass. J Urol. 2009;182(4):1271-9.

2. Wasilijiang W, Xin M, Hong-Zhao L, et al. Evolving renorrhaphy technique for retroperitoneal laparoscopic partial nephrectomy: single-surgeon series. International Journal of Urology Official Journal of the Japanese Urological Association. 2014;21(9):865-73.

3. Zheng JH, Zhang XL, Geng J, et al. Long-term oncologic outcomes of laparoscopic versus open partial nephrectomy. Chin Med J. 2013;126(15):2938-42.

4. Shang JW, Xin MA, Zhang X, et al. Comparison of two different renorrhaphy techniques in retroperitoneal laparoscopic partial nephrectomy for complex tumor. Chin Med J (Engl). 2013;126(24):4629-32.

5. Hervé B, Andrew B, Nimish S, et al. Reducing warm ischaemia time during laparoscopic partial nephrectomy: a prospective comparison of two renal closure techniques. Eur Urol. 2007;52(4):11649.

6. Wang K, Zhang YL, Lin CH, et al. Application of Self-retaining Bidirectional Barbed Absorbable Suture in Retroperito- neoscopic Partial Nephrectomy. International Braz J Urol Official Journal of the Brazilian Society of Urology. 2014;40(2):220-4.

7. Suriano F, Conlin MJ, Buscarini M. Feasibility of felt application for renorrhaphy after laparoscopic partial nephrectomy. Minerva Urologica E Nefrologica. 2014;66(1):83-5.

8. Schauer I, Theimer O, Klatte T, et al. Use of self-retaining barbed sutures decreases cold ischemia time in open nephron-sparing surgery. Wien Klin Wochenschr. 2014;126(11-12):329-34.

9. Shah HN, Nayyar R, Rajamahanty S, et al. Prospective evaluation of unidirectional barbed suture for various indications in surgeon-controlled robotic reconstructive urologic surgery: Wake Forest University experience. International Urology \& Nephrology, 2012, 44(3):775-785.

10. Masumori N, Itoh N, Takahashi S, et al. New technique with combination of felt, Hem-o-lok and Lapra-Ty for suturing the renal parenchyma in laparoscopic partial nephrectomy. Int J Urol. 2012;19(3):273-6.

11. Rosenblatt GS, Fuchs GJ. A comparison of running suture versus figure-8 sutures as the initial step in achieving hemostasis during laparoscopic partial nephrectomy. J Endourol. 2010;24(3):421-4. 
12. Jr W J. Convenient creation and use of suturing supplies for laparoscopic partial nephrectomy. Journal of Endourology, 2009, 23(7):1121-1126.

13. Taneja S, Dakwar GG. Simplified reconstruction after laparoscopic partial nephrectomy using a single-pass suturing technique. J Endourol. 2009;23(4):589-91.

14. Simforoosh N, Tabibi A. Bolsterless laparoscopic partial nephrectomy: a simplification of the technique. J Endourol. 2009;23(6):965-9.

15. Nor Azhari MZ, Tan YH, Sunga PA, et al. Laparoscopic partial nephrectomy for renal tumours: early experience in Singapore general hospital. Annals of the Academy of Medicine Singapore. 2009;38(7):576-80.

16. Nguyen MM, Gill IS. Halving ischemia time during laparoscopic partial nephrectomy. J Urol. 2008;179(2):627-32. discussion 632.

17. Janetschek G. Partial nephrectomy for renal cell carcinoma: trust suturing. J Endourol. 2008;22(9):1933-5.

18. Canales BK, Lynch AC, Fernandes E, et al. Novel Technique of Knotless Hemostatic Renal Parenchymal Suture Repair During Laparoscopic Partial Nephrectomy. Urology. 2007;70(2):358-9.

19. Dinesh A, Paddy O, David C, et al. Modified technique of renal defect closure following laparoscopic partial nephrectomy. Bju International. 2007;100(4):967-70.

20. Wille AH, Maximilian T, Jan R, et al. Laparoscopic partial nephrectomy in renal cell cancer-results and reproducibility by different surgeons in a high volume laparoscopic center. Eur Urol. 2006;49(2):337-43.

21. Seung Hyun J, Saebin J, Hee-Seo S, et al. The Unidirectional Barbed Suture for Renorrhaphy During Laparoscopic Partial Nephrectomy: Stanford Experience. J Laparoendosc Adv Surg Tech. 2013;23(6):521-5.

22. Znaor A, Lortet-Tieulent J, Laversanne M, et al. International Variations and Trends in Renal Cell Carcinoma Incidence and Mortality. Eur Urol. 2015;67(3):519-30.

23. Gandaglia G, Ravi P, Abdollah F, et al. Contemporary incidence and mortality rates of kidney cancer in the United States. Canadian Urological Association journal = Journal de l'Association des urologues du Canada, 2014, 8(7-8):247-252.

24. Thompson RH, Lane BR, Lohse $\mathrm{CM}$, et al. Every minute counts when the renal hilum is clamped during partial nephrectomy. Eur Urol. 2010;58(3):340-5.

25. Link RE, Bhayani SB, Allaf ME, et al. Exploring the learning curve, pathological outcomes and perioperative morbidity of laparoscopic partial nephrectomy performed for renal mass. J Urol. 2005;173(5):1690-4.

\section{Figures}




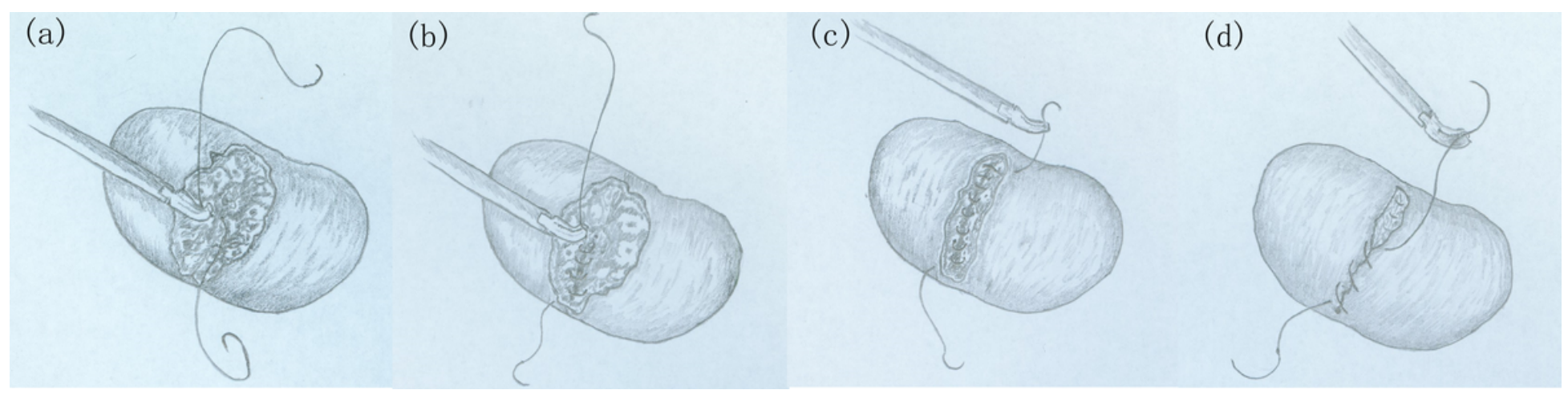

\section{Figure 1}

(a) The needle was run at the mid of the inner tumor bed and the suture was fixed at the midpoint. (b) The needle was run from the midpoint to the bottom throughout the tumor bed with continuous suture. (c) The inner suture was finished and the suture was tightened. (d) The outer layer was closed with continuous suture in the same way as the inner layer. 